AAECC 6, 129-146 (1995)

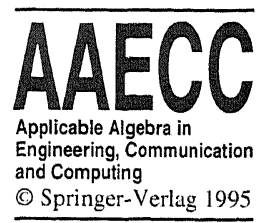

\title{
Incidence and Lattice Calculus with Applications to Stochastic Geometry and Image Analysis ${ }^{\star}$
}

\author{
A. J. Baddeley ${ }^{1}$ and H. J. A. M. Heijmans ${ }^{2}$ \\ 1 Department of Mathematics, University of Western Australia, Nedlands WA 6009, Australia \\ 2 CWI P.O. Box 94079, 1090 GB Amsterdam The Netherlands \\ Received February 17, 1993; revised version January 31, 1994
}

\begin{abstract}
Incidence between subsets is a basic concept of stochastic geometry and mathematical morphology. In this note we discuss a formal generalisation of incidence (and the dual notion of dominance) in the setting of complete lattices. We discuss applications to mathematical morphology, random set theory and combinatorial geometrical probability. We also suggest possible applications to transmission microscopy, digital image discretization and robot motion planning. The generalised incidence structure turns out to be equivalent to the established idea of a lattice adjunction. Using this, many problems in stochastic geometry (BuffonSylvester problem, local knowledge, overprojection effects) can be reformulated as lattice calculations.
\end{abstract}

Keywords: Adjunction, Buffon-Sylvester Problem, Conditional Closure, Discretization, Image Processing, Incidence Relation, Local Knowledge Principle, Mathematical Morphology, Overprojection, Random Closed Sets, Robot Motion Planning, Strong Incidence Functions, Transmission Microscopy, Underprojection.

\section{Introduction}

Two subsets $X, Y \subset \mathbb{R}^{n}$ are 'incident' if $X \cap Y \neq \varnothing$. Incidence is a basic concept of stochastic geometry $[13,24]$. Many classical problems [14, 20,23] concern the probability that a random set will intersect a given fixed set. In the general theory of random sets $[13,16]$, a random subset $X$ of an arbitrary space $S$ is characterized solely by testing whether $X$ intersects $T$ for a sufficiently large class of test sets $T$.

Incidence information $X \cap T \neq \varnothing$ is of course equivalent to information about the partial order of set inclusion $\left(X \cong T\right.$ iff $X \cap T^{c}=\varnothing$ where $T^{c}$ denotes the set complement). In the related field of mathematical morphology [21] recent work $[12,17,18,19,22]$ suggests that the partial order structure is more natural, and enables one to harness the theory of complete lattices $[3,4]$.

^ AMS Mathematics Subject Classification (1985 Revision): 51B05, 60D05, 06A15 
In this note we show that a generalisation of the incidence relation $X \sim Y \Leftrightarrow$ $X \cap Y \neq \varnothing$ is trivially equivalent to the concept of an adjunction in lattice theory. Using this, many problems in stochastic geometry (including the theory of strong incidence functions [13], projection effects [6], and the Buffon-Sylvester problem $[1,2,20])$ can be reformulated as lattice calculations.

General theory is in Sects. 1-3 and applications are discussed in Sects. 4-7. Section 1 recalls some lattice theory; Sect. 2 defines incidence and partial order structures and their (trivial) relationship to adjunctions. In Sect. 3 we go through a calculation in lattice algebra that is found in different incarnations (local knowledge principle, conditional closure) in the applications. Our first application is in Sect. 4 which shows how classical mathematical morphology fits into the lattice setting. In Sect. 5 we show that the non-probabilistic aspects of Kendall's theory of strong incidence functions in the general theory of random sets can be derived from the results in Sects. 1 and 2. In Sect. 6 we use the conditional closure operation of Sect. 3 to handle the Buffon-Sylvester problem. More concrete applications (to projection effects in microscopy, image discretization, and robot motion planning) are described in Sect. 7.

\section{Complete Lattices and Adjunctions}

This section recalls some basic lattice theory [3], in particular the concept of an adjunction $[3,8]$, and results about adjunctions from [12] that are important in the context of mathematical morphology.

A complete lattice is a partially ordered set $(\mathscr{L}, \leqq)$ in which every subset $\mathscr{H} \subseteq \mathscr{L}$ has a supremum and infimum denoted by $\vee \mathscr{H}$ and $\wedge \mathscr{H}$ respectively. In particular there is a greatest element $\mathbf{1}_{\mathscr{L}}$ and a least element $\mathbf{0}_{\mathscr{L}}$. A complete lattice is Boolean if suprema distribute over infima and vice versa, and if every $X \in \mathscr{L}$ has a unique complement $X^{*}$ such that $X \vee X^{*}=\mathbf{1}_{\mathscr{L}}, X \wedge X^{*}=\mathbf{0}_{\mathscr{L}}$.

For example the class $\mathscr{P}(E)$ of all subsets of an arbitrary set $E$, ordered by set inclusion $X \leqq Y \Leftrightarrow X \subseteq Y$, is a complete Boolean lattice whose supremum, infimum and complement operations are equivalent to set union, intersection, and complement in $E$ respectively. The closed subsets of a topological space form a complete lattice, where infimum is set intersection and supremum is the topological closure of the set union.

Definition 1. Let $\mathscr{M}, \mathscr{L}$ be complete lattices and let $\varepsilon: \mathscr{M} \rightarrow \mathscr{L}$ and $\delta: \mathscr{L} \rightarrow \mathscr{M}$. The pair $(\varepsilon, \delta)$ is called an adjunction between $\mathscr{M}$ and $\mathscr{L}$ if for every $X \in \mathscr{L}$ and $Y \in \mathscr{M}$,

$$
\delta(X) \leqq Y \Leftrightarrow X \leqq \varepsilon(Y) .
$$

An example of a nontrivial adjunction is $\mathscr{L}=$ open sets, $\mathscr{M}=$ closed sets of a topological space, $\delta(X)=\operatorname{cl}(X)=$ topological closure of $X, \varepsilon(Y)=\operatorname{int}(Y)=$ interior of $Y$.

Let $\mathscr{L}, \mathscr{M}$ be complete lattices. The identity mapping on $\mathscr{L}$ is denoted by id . A mapping $\psi: \mathscr{L} \rightarrow \mathscr{M}$ is called increasing if $X \leqq X^{\prime}$ implies that $\psi(X) \leqq \psi\left(X^{\prime}\right)$. We say that $\psi$ is a dilation if $\psi$ distributes over suprema, that is for an arbitrary collection $\left\{X_{i} \mid i \in I\right\}$,

$$
\psi\left(\bigvee_{i}^{\vee} X_{i}\right)=\bigvee_{i} \psi\left(X_{i}\right)
$$


Taking $I$ to be empty gives $\psi(\mathbf{0})=\mathbf{0}$. Dually, $\psi$ is called an erosion if it distributes over infima,

$$
\psi\left(\wedge_{i} X_{i}\right)=\bigwedge_{i} \psi\left(X_{i}\right)
$$

Taking $I$ empty gives $\psi(\mathbf{1})=\mathbf{1}$. It is obvious that dilations and erosions are increasing mappings.

Proposition 1. Let $\mathscr{M}, \mathscr{L}$ be complete lattices.

(a) If $(\varepsilon, \delta)$ is an adjunction between $\mathscr{M}$ and $\mathscr{L}$, then $\delta$ is a dilation and $\varepsilon$ an erosion.

(b) To every erosion $\varepsilon: \mathscr{M} \rightarrow \mathscr{L}$ there corresponds a unique dilation $\delta: \mathscr{L} \rightarrow \mathscr{M}$ such that $(\varepsilon, \delta)$ is an adjunction.

(c) To every dilation $\delta: \mathscr{L} \rightarrow \mathscr{M}$ there corresponds a unique erosion $\varepsilon: \mathscr{M} \rightarrow \mathscr{L}$ such that $(\varepsilon, \delta)$ is an adjunction.

For proofs of (a)-(c) see [12], Proposition 2.5, Theorem 2.7 (ii) and (i) respectively. If $\varepsilon: \mathscr{M} \rightarrow \mathscr{L}$ is an erosion then, trivially, the adjoint dilation $\delta$ is given by

$$
\delta(X)=\wedge\{Y \in \mathscr{M} \mid \delta(X) \leqq Y\}=\wedge\{Y \in \mathscr{M} \mid X \leqq \varepsilon(Y)\} .
$$

A similar expression holds for $\varepsilon$ in terms of $\delta$.

A mapping $\psi: \mathscr{L} \rightarrow \mathscr{L}$ is called a closing if it is increasing, extensive $\left(\psi \geqq\right.$ id $\left._{\mathscr{L}}\right)$ and idempotent $\left(\psi^{2}=\psi\right)$. If $\psi$ is increasing, anti-extensive $\left(\psi \leqq \mathbf{i d}_{\mathscr{L}}\right)$ and idempotent, then it is called an opening.

Proposition 2. Let $(\varepsilon, \delta)$ be an adjunction between $\mathscr{M}$ and $\mathscr{L}$. Then

(a) $\varepsilon \delta \geqq$ id $_{\mathscr{L}}$ and $\delta \varepsilon \leqq \mathbf{i d}_{\mathscr{M}}$;

(b) $\delta \varepsilon \delta=\delta$ and $\varepsilon \delta \varepsilon=\varepsilon$;

(c) $\varepsilon \delta$ is a closing on $\mathscr{L}$ and $\delta \varepsilon$ is an opening on $\mathscr{M}$.

This is trivial, but see [12], Propositions 2.6 and 2.8 .

We say that $X \in \mathscr{L}$ is closed with respect to $(\varepsilon, \delta)$ if $\varepsilon \delta(X)=X$. An element $Y \in \mathscr{M}$ is said to be open with respect to $(\varepsilon, \delta)$ if $\delta \varepsilon(Y)=Y$.

Proposition 3. Let $(\varepsilon, \delta)$ be an adjunction between $\mathscr{M}$ and $\mathscr{L}$.

(a) $X \in \mathscr{L}$ is closed if and only if $X=\varepsilon(Y)$ for some $Y \in \mathscr{M}$.

(b) $Y \in \mathscr{M}$ is open if and only if $Y=\delta(X)$ for some $X \in \mathscr{L}$.

(c) Arbitrary infima of closed elements are closed; arbitrary suprema of open elements are open.

(d) $\varepsilon \delta(X)$ is the smallest closed element $\geqq X ; \delta \varepsilon(Y)$ is the largest open element $\leqq Y$.

Proof. If $X$ is closed then $X=\varepsilon \delta(X)$ and hence $X=\varepsilon(Y)$ if one puts $Y=\delta(X)$. Conversely, if $X=\varepsilon(Y)$, then $\varepsilon \delta(X)=\varepsilon \delta \varepsilon(Y)=\varepsilon(Y)=X$ by Proposition 2(b). This proves (a), and (b) is proved similarly. For (c), let $X_{i} \in \mathscr{L}$ be closed; then $X_{i}=\varepsilon\left(Y_{i}\right)$ for some $Y_{i}$, hence $\wedge_{i} X_{i}=\wedge_{i} \varepsilon\left(Y_{i}\right)=\varepsilon\left(\wedge_{i} Y_{i}\right)$. To prove (d), first note that $\varepsilon \delta(X)$ is closed and $\geqq X$ by Proposition 2 . For $Y$ closed and $\geqq X$ we have $Y=\varepsilon \delta(Y) \geqq \varepsilon \delta(X)$ since $\varepsilon, \delta$ are increasing. The second statement follows similarly.

Proposition 4. Let $Y \in \mathscr{M}$ be open. Then for $X \in \mathscr{L}$

$$
\delta(X)=Y
$$


iff

$$
\varepsilon \delta(X)=\varepsilon(Y) .
$$

One solution of (2) is $X=\varepsilon(Y)$.This is the largest solution and the unique closed solution.

This follows from Propositions 2(c) and 3.

If both $\mathscr{L}$ and $\mathscr{M}$ are complete Boolean lattices, and if $\psi: \mathscr{L} \rightarrow \mathscr{M}$, then we can define the dual mapping $\psi^{*}: \mathscr{L} \rightarrow \mathscr{M}$ by

$$
\psi^{*}(X)=\left[\psi\left(X^{*}\right)\right]^{*} .
$$

Proposition 5. Let $(\varepsilon, \delta)$ be an adjunction between the complete Boolean lattices $\mathscr{M}$ and $\mathscr{L}$. Then $\left(\delta^{*}, \varepsilon^{*}\right)$ is an adjunction between $\mathscr{L}$ and $\mathscr{M}$. An element $X \in \mathscr{L}$ is closed with respect to $(\varepsilon, \delta)$ iff $X^{*}$ is open with respect to $\left(\delta^{*}, \varepsilon^{*}\right)$.

Proof. For $X \in \mathscr{L}, Y \in \mathscr{M}$ we have $\varepsilon^{*}(Y) \leqq X$ iff $X^{*} \leqq \varepsilon\left(Y^{*}\right)$ iff $\delta\left(X^{*}\right) \leqq Y^{*}$ iff $Y \leqq \delta^{*}(X)$, so that $\left(\delta^{*}, \varepsilon^{*}\right)$ is an adjunction. Suppose that $X \in \mathscr{L}$ is closed with respect to $(\varepsilon, \delta)$; then

$$
\varepsilon^{*} \delta^{*}\left(X^{*}\right)=\varepsilon^{*}\left([\delta(X)]^{*}\right)=[\varepsilon \delta(X)]^{*}=X^{*}
$$

so that $X^{*}$ is open with respect to $(\varepsilon, \delta)$. The converse is proved dually.

\section{Incidence and Dominance Structures}

This section defines generalisations of the concepts of incidence and dominance, and shows that they are broadly equivalent to lattice adjunctions.

Definition 2. Let $\mathscr{L}, \mathscr{M}$ be complete lattices. $A$ dominance relation $\leftrightharpoons$ is a relation on $\mathscr{L} \times \mathscr{M}$ such that for any collections $\left\{X_{i} \mid i \in I\right\} \cong \mathscr{L},\left\{Y_{j} \mid j \in J\right\} \cong \mathscr{M}$ where $I, J$ are arbitrary index sets,

$$
\bigvee_{i} X_{i} \preceq \wedge_{j} Y_{j} \Leftrightarrow \forall i, j\left(X_{i} \preceq Y_{j}\right)
$$

For example with $\mathscr{L}=\mathscr{M}=\mathscr{P}(S)$ for an arbitrary space $S$,

$$
X \preceq Y \text { iff } X \cong Y
$$

is a dominance relation. A dominance relation is never void since

$$
0_{\mathscr{L}} \preceq Y(Y \in \mathscr{M}) \text { and } X \preceq \mathbf{1}_{\mathscr{M}}(X \in \mathscr{L})
$$

(taking $I$ or $J$ respectively to be empty) and it has the transitivity property that if $X \preceq Y, X^{\prime} \leqq X$ and $Y \leqq Y^{\prime}$ then $X^{\prime} \preceq Y^{\prime}$. (For then $X^{\prime} \vee X=X \preceq Y=Y \wedge Y^{\prime}$ implies $X^{\prime} \preceq Y^{\prime}$ by definition).

Theorem 1. If $(\varepsilon, \delta)$ is an adjunction between $\mathscr{M}$ and $\mathscr{L}$, then $\preceq$ defined by

$$
X \preceq Y \Leftrightarrow \delta(X) \leqq Y \Leftrightarrow X \leqq \varepsilon(Y)
$$

is a dominance relation. Conversely if $\preceq$ is a dominance relation on $\mathscr{L} \times \mathscr{M}$ then there exists a unique adjunction $(\varepsilon, \delta)$ between $\mathscr{M}$ and $\mathscr{L}$ for which (4) holds, namely

$$
\begin{aligned}
& \delta(X)=\wedge\{Y \mid X \preceq Y\}, \\
& \varepsilon(Y)=\vee\{X \mid X \preceq Y\} .
\end{aligned}
$$


Proof. Let $(\varepsilon, \delta)$ be an adjunction and define $\preceq$ by (4). Suppose $\vee_{i} X_{i} \preceq \wedge_{j} Y_{j}$; then $\vee_{i} \delta\left(X_{i}\right)=\delta\left(\vee_{i} X_{i}\right) \leqq \wedge_{j} Y_{j}$. By definition of $\wedge$ and $\vee$ it follows that $\bar{\delta}\left(X_{i}\right) \leqq Y_{j}$ for every $i, j$. Thus $X_{i} \leq Y_{j}$ for all $i, j$. The converse follows a similar argument.

Let $\preceq$ be a dominance relation and $\delta, \varepsilon$ the maps constructed in (5)-(6). By definition of $\wedge$ if $X \preceq Y$ then $\delta(X) \leqq Y$; conversely if $\delta(X) \leqq Y$ then $X \preceq Y$ by the transitivity property stated above. Similarly for $\varepsilon$. That is, $(\varepsilon, \delta)$ is an adjunction. The adjunction is unique, since any adjunction satisfying (4) must be of the form (5)-(6) since e.g. $\delta(X)=\wedge\{Y \mid \delta(X) \leqq Y\}=\wedge\{Y \mid X \preceq Y\}$.

Definition 3. An incidence relation $\sim$ is a relation on $\mathscr{L} \times \mathscr{M}$ such that for arbitrarily large collections $\left\{X_{i} \mid i \in I\right\} \cong \mathscr{L},\left\{Y_{j} \mid j \in J\right\} \subseteq \mathscr{M}$,

$$
\bigvee_{i} X_{i} \sim \underset{j}{\vee} Y_{j} \Leftrightarrow \exists i, j\left(X_{i} \sim Y_{j}\right)
$$

Taking $I$ or $J$ to be empty gives

$$
\mathbf{0}_{\mathscr{L}} \nsucc Y(Y \in \mathscr{M}) \text { and } X \neq \mathbf{0}_{\|}(X \in \mathscr{L}) \text {. }
$$

The standard example is $X \sim Y \Leftrightarrow X \cap Y \neq \varnothing$ for subsets $X, Y$ of an arbitrary space. The attraction of incidence relations is that they are intuitively easier to define, and the symmetry of $\mathscr{L}, \mathscr{M}$ in the definition is a simple expression of projective duality, $X$ hits $Y$ iff $Y$ hits $X$.

Theorem 2. If $\mathscr{M}$ is Boolean, an incidence relation $\sim$ on $\mathscr{L} \times \mathscr{M}$ is equivalent to a dominance relation $\preceq$ on $\mathscr{L} \times \mathscr{M}$ through

$$
X \preceq Y \Leftrightarrow X \nsim Y^{*} .
$$

The proof is trivial. In this case the associated dilation and erosion can be expressed as

$$
\begin{aligned}
& \delta(X)=[\vee\{Y \in \mathscr{M} \mid X \nsucc Y\}]^{*} \\
& \varepsilon(Y)=\vee\left\{X \in \mathscr{L} \mid X \nsucc Y^{*}\right\} .
\end{aligned}
$$

Remark. Even if $\mathscr{L}, \mathscr{M}$ are not Boolean, a dominance relation on $\mathscr{L} \times \mathscr{M}$ corresponds to an incidence relation on $\mathscr{L} \times \mathscr{M}^{\prime}$ where $\mathscr{M}^{\prime}$ is the dual lattice of $\mathscr{M}$ (i.e. with order reversed).

Suppose we have two Boolean complete lattices $\mathscr{L}, \mathscr{M}$ and an incidence relation $\sim$ on $\mathscr{L} \times \mathscr{M}$. This generates two dual adjunctions,

$$
\begin{aligned}
\delta(X) & =[\vee\{Y \in \mathscr{M} \mid X \nsucc Y\}]^{*} \\
\varepsilon(Y) & =\vee\left\{X \in \mathscr{L} \mid X \nsucc Y^{*}\right\} \\
\delta^{*}(X)=\delta\left(X^{*}\right)^{*} & =\vee\left\{Y \in \mathscr{M} \mid X^{*} \nsucc Y\right\} \\
\varepsilon^{*}(Y)=\varepsilon\left(Y^{*}\right)^{*} & =[\vee\{X \in \mathscr{L} \mid X \nsucc Y\}]^{*}
\end{aligned}
$$

which in most cases are not identical. The analogues of (5)-(6) are

$$
\begin{aligned}
\delta^{*}(X) & =\vee\left\{Y \mid X^{*} \preceq Y^{*}\right\} \\
\varepsilon^{*}(Y) & =\wedge\left\{X \mid X^{*} \preceq Y^{*}\right\} .
\end{aligned}
$$




\section{Local Knowledge and Conditional Closing}

Throughout this section we assume $\mathscr{L}, \mathscr{M}$ are complete Boolean lattices equipped with an incidence relation $\sim$.

Many calculations turn out to be expressible in the following context.

Definition 4. Fix $N \in \mathscr{M}$ and let

$$
\mathscr{M}_{N}=\{M \in \mathscr{M} \mid M \leqq N\}
$$

with the inherited order relation. This is a complete Boolean lattice, with complement operation

$$
c_{N}(Y)=Y^{*} \wedge N .
$$

Lemma 1. The restriction of the incidence relation $\sim$ to $\mathscr{L} \times \mathscr{M}_{N}$ is an incidence relation; its associated adjunction is

$$
\begin{aligned}
& \delta_{N}(X)=\delta(X) \wedge N \\
& \varepsilon_{N}(Y)=\varepsilon\left(Y \vee N^{*}\right) .
\end{aligned}
$$

Proof. The associated dominance relation is clearly

$$
X \preceq_{N} Y \Leftrightarrow X \nsucc\left(Y^{*} \wedge N\right) .
$$

By equations (7-8)

$$
\begin{aligned}
\delta_{N}(X) & =c_{N}\left(\vee\left\{Y \in \mathscr{M}_{N} \mid X \ngtr Y\right\}\right) \\
& =c_{N}(\vee\{Y \in \mathscr{M} \mid X \nsucc Y\} \wedge N) \\
& =\left(\delta(X)^{*} \wedge N\right)^{*} \wedge N \\
& =\left(\delta(X) \vee N^{*}\right) \wedge N \\
& =\delta(X) \wedge N \\
\varepsilon_{N}(Y) & =\vee\left\{X \in \mathscr{L} \mid X \nsucc\left(Y^{*} \wedge N\right)\right\} \\
& =\vee\left\{X \in \mathscr{L} \mid X \nsucc\left(Y \vee N^{*}\right)^{*}\right\} \\
& =\varepsilon\left(Y \vee N^{*}\right) . \quad \square
\end{aligned}
$$

Since $\left(\varepsilon_{N}, \delta_{N}\right)$ is an adjunction between $\mathscr{M}_{N}$ and $\mathscr{L}$, the results of Sect. 1 apply. For example the associated closing operator is

$$
\begin{aligned}
\varepsilon_{N} \delta_{N}(X) & =\varepsilon\left((\delta(X) \wedge N) \vee N^{*}\right) \\
& =\varepsilon\left(\delta(X) \vee N^{*}\right) .
\end{aligned}
$$

Notice that $\varepsilon_{N} \delta_{N}(X)$ is the largest solution $W$ in $\mathscr{L}$ of

$$
\delta(W) \wedge N=\delta(X) \wedge N .
$$

Since we are restricted to elements of $\mathscr{M}$ which are $\leqq N$ we call $\varepsilon_{N} \delta_{N}$ a conditional closing. We now specialise this to the case where $N$ is of the form $N=\delta(Z)$ for some $Z \in \mathscr{L}$.

Proposition 6. For fixed $X, Z \in \mathscr{L}$ the largest solution $W$ of

$$
\delta(W) \wedge \delta(Z)=\delta(X) \wedge \delta(Z)
$$


is

$$
W=\varepsilon\left(\delta(X) \vee \delta(Z)^{*}\right)
$$

Proof. Setting $N=\delta(Z)$ in Lemma 1 we recognise (16) as the equation $\delta_{N}(W)=$ $\delta_{N}(X)$. By Proposition 4 the largest solution is $W=\varepsilon_{N}\left(\delta_{N}(X)\right)$. But this is $W=$ $\varepsilon\left((\delta(X) \wedge N) \vee N^{*}\right)=\varepsilon\left(\delta(X) \vee \delta(Z)^{*}\right)$

We give an example of the conditional closing in Sect. 6.

Consider an element $Z \in \mathscr{L}$. Think of $Z$ as a window which bounds the objects which we are able to perceive; that is, assume that for any element $X \in \mathscr{L}$ we only have information about the part $X \wedge Z$. From this local knowledge of $X$ it is still possible to compute $\delta(X)$ inside a window $W \in \mathscr{M}$. The next result is dubbed the "local knowledge principle" after a result in mathematical morphology [21, pp. 11, $49,62]$.

Proposition 7. Fix $Z \in \mathscr{L}$. Then the largest element $W \in \mathscr{M}$ satisfying

$$
\delta(X) \wedge W=\delta(X \wedge Z) \wedge W \text { for all } X \in \mathscr{L}
$$

is $W=\delta^{*}(Z)$.

Proof. Set $N=\delta^{*}(Z)$ in Lemma 1 ; the identity $\delta_{N} \varepsilon_{N} \delta_{N}=\delta_{N}$ of Proposition 2(b) reads

$$
\begin{aligned}
\delta(X) \wedge \delta^{*}(Z) & =\delta_{N}(X)=\delta_{N} \varepsilon_{N} \delta_{N}(X) \\
& =\delta\left(\varepsilon\left[\left(\delta(X) \wedge \delta^{*}(Z)\right) \vee \delta\left(Z^{*}\right)\right]\right) \wedge \delta^{*}(Z) \\
& =\delta\left(\varepsilon\left(\delta(X) \vee \delta\left(Z^{*}\right)\right)\right) \wedge \delta^{*}(Z) \\
& =\delta \varepsilon \delta\left(X \vee Z^{*}\right) \wedge \delta^{*}(Z) \\
& =\delta\left(X \vee Z^{*}\right) \wedge \delta^{*}(Z) .
\end{aligned}
$$

Using the identity $X \vee Z^{*}=(X \wedge Z) \vee Z^{*}$ and the distributivity of $\delta$ over suprema, this becomes

$$
\begin{aligned}
{\left[\delta(X \wedge Z) \vee \delta\left(Z^{*}\right)\right] \wedge \delta^{*}(Z) } & =\left[\delta(X \wedge Z) \wedge \delta^{*}(Z)\right] \vee\left[\delta\left(Z^{*}\right) \wedge \delta^{*}(Z)\right] \\
& =\delta(X \wedge Z) \wedge \delta^{*}(Z)
\end{aligned}
$$

This gives the identity in (17) for fixed $X, Z$. Since $W=\delta^{*}(Z)$ does not depend on $X$ the identity is true for all $X$.

By Proposition 4, $W=\delta^{*}(Z)$ is the largest element satisfying the identity in (17) for fixed $X, Z$. Again since $W$ does not depend on $X$ it is the largest element satisfying (17).

Examples of the local knowledge principle will be given in the ensuing sections. There is also a dual identity

$$
\varepsilon^{*}(Y) \wedge \varepsilon(Z)=\varepsilon^{*}(Y \wedge Z) \wedge \varepsilon(Z)
$$

in which $\varepsilon(Z)$ is the smallest $W$ for which this identity holds.

\section{Application to Mathematical Morphology}

Mathematical morphology is a geometrical approach to quantitative image analysis based on set-theoretical operations such as Minkowski sum and difference. For a 
comprehensive treatment we refer to $[16,21]$. Recently it has been shown $[11,12$, $17,18,19,22]$ that mathematical morphology can be extended to arbitrary complete lattices. Adjunctions play a crucial role in this abstract formulation. In this section we show for illustration how the notions of incidence can be use to formalise classical Euclidean morphology.

\subsection{Classical Euclidean Morphology}

Define the translation of a subset $A$ by a vector $x \in \mathbb{R}^{n}$ to be

$$
A_{x}=\{a+x \mid a \in A\} .
$$

For subsets $X, A \subseteq \mathbb{R}^{n}$ define the Minkowski sum

$$
X \oplus A=\bigcup_{a \in A} X_{a}=\{x+a \mid x \in X, a \in A\}
$$

and Minkowski difference

$$
X \ominus A=\bigcap_{a \in A} X_{-a}
$$

We can also write

$$
\begin{aligned}
& X \oplus A=\left\{z \in \mathbb{R}^{n} \mid \tilde{A}_{z} \cap X \neq \varnothing\right\} \\
& X \ominus A=\left\{z \in \mathbb{R}^{n} \mid A_{z} \leqq X\right\}
\end{aligned}
$$

where $\check{A}$ is the reflection of $A$ through 0 ,

$$
\check{A}=\{-a \mid a \in A\} \text {. }
$$

The closing and opening of $X$ by $A$ are defined as

$$
\begin{aligned}
& X \bullet A=(X \oplus A) \ominus A \\
& X \circ A=(X \ominus A) \oplus A
\end{aligned}
$$

respectively.

The closing $X \mapsto X \bullet A$ is

(i) increasing, i.e. $X \cong Y \Rightarrow X \bullet A \subseteq Y \bullet A$;

(ii) idempotent, i.e. $(X \bullet A) \bullet A=X \bullet A$;

(iii) extensive, i.e. $X \cong X \bullet A$.

See [21, pp. 52ff]. The opening operator also satisfies statements (i)-(ii) while (iii) is replaced by anti-extensivity, $X_{\circ} A \cong X$.

\subsection{Lattice Reformulation}

Let $\mathscr{L}=\mathscr{M}=\mathscr{P}\left(\mathbb{R}^{n}\right)$. Fix a subset $A \cong \mathbb{R}^{n}$ and define for $X, Y \cong \mathbb{R}^{n}$

$$
X \sim Y \Leftrightarrow A_{x} \cap Y \neq \varnothing \text { for some } x \in X ;
$$

this is clearly an incidence relation; note that it is not symmetric in $X, Y$ but

$$
X \sim Y \Leftrightarrow X \cap \check{A}_{y} \neq \varnothing \text { for some } y \in Y .
$$


The associated dominance relation is

We have

$$
X \preceq Y \Leftrightarrow A_{x} \subseteq Y \text { for all } x \in X .
$$

and

$$
\begin{aligned}
\delta(\mathrm{X}) & =\bigcap\{Y \mid X \preceq Y\} \\
& =\bigcap\left\{Y \mid \forall x \in X, A_{x} \subseteq Y\right\} \\
& =\bigcup_{x \in X} A_{x} \\
& =X \oplus A
\end{aligned}
$$

$$
\begin{aligned}
\varepsilon(Y) & =\bigcup\{X \mid X \preceq Y\} \\
& =\left\{x \mid A_{x} \leqq Y\right\} \\
& =Y \ominus A .
\end{aligned}
$$

It follows that $\varepsilon \delta(X)=X \bullet A$ and $\delta \varepsilon(X)=X \circ A$.

In mathematical morphology $\left[21\right.$, p. 53] a set $X \subseteq \mathbb{R}^{n}$ is called $A$-closed if $X \bullet A=X$. Note that this definition coincides with that given in Sect. 1. In particular we find that a set $X$ is $A$-closed iff $X$ is of the form $X=Y \oplus A$ for some $Y \subseteq \mathbb{R}^{n}$.

The dual adjunction is

$$
\begin{aligned}
\delta^{*}(X) & =\bigcup\left\{Y \mid X^{c} \nsucc Y\right\} \\
& =Y \ominus \check{A} \\
\varepsilon^{*}(Y) & =(\bigcup\{X \mid X \nsucc Y\})^{c} \\
& =Y \oplus \check{A} .
\end{aligned}
$$

The algebraic properties of the closing operator listed in (i)-(iii) of the previous subsection follow immediately from Proposition 2.

In this case the local knowledge principle stated in Proposition 7 reads as

$$
(X \oplus A) \cap(Z \ominus \check{A})=((X \cap Z) \oplus A) \cap(Z \ominus \check{A})
$$

for any $X, Z, A \subseteq \mathbb{R}^{n}$. This means that if a set $X$ is only observed within a window $Z$, the dilation $X \oplus A$ can only be computed within the reduced window $Z \ominus \check{A}$. We refer to [21, pp. $11,49,62]$ for similar statements.

The dual identity (18) is a similar statement with $A$ and $\breve{A}$ exchanged.

\section{Random Set Theory}

\subsection{Strong Incidence Functions}

Kendall [13] introduced the following concepts. Let $S$ be an arbitrary nonempty set, and $\mathscr{T} \subset \mathscr{P}(S)$ an arbitrary class of nonempty subsets of $S$ (called 'traps') that cover $S$. The incidence function of $X$ over $\mathscr{T}$ is the function $I_{X}: \mathscr{T} \rightarrow\{0,1\}$ defined by

$$
I_{X}(T)= \begin{cases}1 & \text { if } X \cap T \neq \varnothing \\ 0 & \text { else }\end{cases}
$$


The goal was to construct random sets as random 0,1 -valued functions on $\mathscr{T}$; hence one needs to determine when an arbitrary function $f: \mathscr{T} \rightarrow\{0,1\}$ is the incidence function of some subset $X$, and in that case, to find all solutions $X$. That is, to solve for $X$ in

$$
f=I_{X} .
$$

Suppose $X$ is a solution. Then $f(T)=0$ implies $X \cap T=\varnothing$, or equivalently, $X \cong T^{c}$. This yields

$$
X \cong \bigcap\left\{T^{c} \mid T \in \mathscr{T} \quad \text { and } \quad f(T)=0\right\}=(\bigcup\{T \mid T \in \mathscr{T} \quad \text { and } \quad f(T)=0\})^{c} .
$$

The following is a paraphrase of results in [13].

Definition 5. The $\mathscr{T}$-support of an arbitrary function $f: \mathscr{T} \rightarrow\{0,1\}$ is the set

$$
\operatorname{spt}(f, \mathscr{T})=(\bigcup\{T \in \mathscr{T} \mid f(T)=0\})^{c} .
$$

The $\mathscr{T}$-closure of an arbitrary set $X \cong S$ is

$$
\operatorname{clos}(X, \mathscr{T})=\operatorname{spt}\left(I_{X}, \mathscr{T}\right)=(\bigcup\{T \in \mathscr{T} \mid T \cap X=\varnothing\})^{c} .
$$

We say that $X$ is $\mathscr{T}$-closed if $X=\operatorname{clos}(X, \mathscr{T})$.

Definition 6. A function $f: \mathscr{T} \rightarrow\{0,1\}$ is a strong incidence function (s.i.f.) if

$$
T \leqq \bigcup_{i} T_{i} \text { implies } f(T) \leqq \max _{i} f\left(T_{i}\right)
$$

for arbitrarily large collections $\left\{T_{i} \mid i \in I\right\}$.

Obviously every incidence function $I_{X}$ is a strong incidence function. Conversely.

Theorem 3 (Kendall) $A$ function $f: \mathscr{T} \rightarrow\{0,1\}$ can be written in the form $f=I_{X}$ iff it is a strong incidence function. In that case, one solution is $X=\mathbf{s p t}(f, \mathscr{T})$. This is the largest solution, and the unique $\mathscr{T}$-closed solution. The other solutions $Y$ are precisely those sets for which $\operatorname{clos}(Y, \mathscr{T})=X$.

The theorem is established in [13] by deducing a number of properties of the $\mathscr{T}$-support and the $\mathscr{T}$-closure.

\subsection{Lattice Reformulation}

Let $S, \mathscr{T}$ be as in the previous subsection. We will show how the constructions described in Sects. 1-2 'automatically' produce the operators of strong incidence function theory.

Let $\mathscr{L}=\mathscr{P}(S)$ with the partial order of set inclusion, and let $\mathscr{M}$ be the complete Boolean lattice of all functions $f: \mathscr{T} \rightarrow\{0,1\}$ with pointwise order $f \leqq f^{\prime} \Leftrightarrow f(T) \leqq$ $f^{\prime}(T)$ for all $T$. Of course $\mathscr{M}$ could be identified with $\mathscr{P}(\mathscr{T})$ via the correspondence $f \leftrightarrow\{T \in \mathscr{T} \mid f(T)=1\}$.

Define an incidence relation by

$$
X \sim f \Leftrightarrow \exists T \in \mathscr{T}(X \cap T \neq \varnothing \text { and } f(T)=1) .
$$

The associated dominance relation is

$$
X \preceq f \Leftrightarrow X \ngtr 1-f
$$




$$
\begin{aligned}
& \Leftrightarrow \nexists T \in \mathscr{T}(f(T)=0 \text { and } X \cap T \neq \varnothing) \\
& \Leftrightarrow \forall T \in \mathscr{T}(X \cap T \neq \varnothing \Rightarrow f(T)=1) .
\end{aligned}
$$

Applying Theorem 1, the associated adjunction has

$$
\delta(X)=\wedge\{f \mid X \preceq f\}=\wedge\{f \mid \forall T \in \mathscr{T}, X \cap T \neq \varnothing \Rightarrow f(T)=1\}
$$

i.e. this is the function on $\mathscr{T}$ with $f(T)=1$ iff $X \cap T \neq \varnothing$. In other words, $\delta(X)=I_{X}$ is Kendall's incidence function of $X$. Further

$$
\begin{aligned}
\varepsilon(f) & =\bigcup\{X \mid X \preceq f\} \\
& =\bigcup\{X \mid \forall T \in \mathscr{T}, X \cap T \neq \varnothing \Rightarrow f(T)=1\} \\
& =\bigcap\left\{T^{c} \mid T \in \mathscr{T}, f(T)=0\right\} \\
& =\operatorname{spt}(f, \mathscr{T}) .
\end{aligned}
$$

The associated closing is

$$
\varepsilon \delta(X)=\operatorname{spt}\left(I_{X}, \mathscr{T}\right)=\operatorname{clos}(X, \mathscr{T}),
$$

i.e. $\varepsilon \delta$ is Kendall's $\mathscr{T}$-closure operator, see Definition 5. On the other hand

$$
\delta \varepsilon(f)=I_{\mathbf{s p t}(f, \mathscr{T})}
$$

is the incidence function of the support of $f$; this is the largest strong incidence function below $f$. Hence $\delta \varepsilon$ coincides with the operator $\mathbf{S}$ of Kendall [13, thm 7, p. 334].

A set $X$ is closed under the adjunction, $\varepsilon \delta(X)=X$, iff it is a $\mathscr{T}$-closed set in Kendall's sense (Definition 5). By Proposition 3(a) this is equivalent to $X=\varepsilon(f)=$ $\operatorname{spt}(f, \mathscr{T})$ for some $f$. that is

A function $f$ is open under the adjunction, $\delta \varepsilon(f)=f$, if and only if $f=I_{\mathbf{s p t}(f, \mathscr{F})}$,

$$
f(T)=1 \Leftrightarrow T \cap \operatorname{spt}(f, \mathscr{T}) \neq \varnothing
$$

i.e.

$$
f(T)=0 \Leftrightarrow T \leqq \bigcup_{f\left(T^{\prime}\right)=0} T^{\prime}
$$

i.e. $f$ is a strong incidence function in the sense of Kendall. Theorem 3 then follows from Propositions 3(b) and 4.

In the dual adjunction, $\delta^{*}(X)$ is a 'containment function'

$$
\left[\delta^{*}(X)\right](T)= \begin{cases}1 & \text { if } T \subseteq X \\ 0 & \text { else }\end{cases}
$$

while $\varepsilon^{*}$ could be called the 'trace' operator

$$
\varepsilon^{*}(f)=\bigcup_{f(T)=1} T .
$$

The dual opening is thus the " $\mathscr{T}$-interior"

$$
\varepsilon^{*} \delta^{*}(X)=\bigcup\{T \mid T \leqq X\}
$$

The local knowledge principle (Proposition 7) states that for given $Z \cong S$ is the 
largest set of traps $W \cong \mathscr{T}$ satisfying

$$
I_{X} \equiv I_{X \cap Z} \quad \text { on } W
$$

for all $X \subseteq S$, is $W=\delta^{*}(Z)$. In other words, if $X$ is an unknown set but $X \cap Z$ is known, then the incidence function of $X$ is known over the class of traps $T$ satisfying $T \leqq Z$ (and not over any larger class, in general).

The dual principle (18) states that for $Z \subseteq \mathscr{T}$ given, spt $(Z, \mathscr{T})$ is the largest subset of $S$ on which the trace of $Y$ coincides with the trace of $Y \cap Z$ for all $Y \cong \mathscr{T}$. In other words if $f: \mathscr{T} \rightarrow\{0,1\}$ is an unknown function whose values are known only on $Z \cong \mathscr{T}$, then the trace of $f$ can be reconstructed within $\operatorname{spt}(Z, \mathscr{T})$.

\section{Buffon-Sylvester Problem}

\subsection{Description}

Let $\Lambda$ be the set of all infinite straight lines in $\mathbb{R}^{2}$. For $A \subset \mathbb{R}^{2}$ define

$$
[A]=\{\ell \in \Lambda \mid \ell \cap A \neq \varnothing\} .
$$

and let $1_{[A]}: \Lambda \rightarrow\{0,1\}$ be its indicator function

$$
1_{[A]}(\ell)= \begin{cases}1 & \text { if } \ell \in[A] \\ 0 & \text { else }\end{cases}
$$

The Buffon-Sylvester problem [1,2], [14, pp. 60-61], [20, pp. 27-34], [23, pp. 65-70] concerns the probabilities of events $[A]$ and finite combinations $[A] \cap[B],[A] \cup[B]$, etc under a probability distribution $P$ on $\Lambda$. The measure $P([A])$ is relatively straightforward to compute for convex compact $A$ (see [2]). Sylvester [25] introduced the following arguments:

(1) if $X \subset \mathbb{R}^{2}$ is compact and path-connected, then $[X]=[\operatorname{co} X]$ where $\operatorname{co} X$ is the convex hull of $X$.

(2) If $A, B \subset \mathbb{R}^{2}$ are compact convex sets with $A \cap B \neq \varnothing$, then

$$
1_{[A] \cap[B]}=1_{[A]}+1_{[B]}-1_{[\mathbf{c o}(A \cup B)]}
$$

so that $P([A] \cap[B])=P([A])+P([B])-P(\operatorname{co}(A \cup B))$ for any $P$; and

(3) If $A, B \subset \mathbb{R}^{2}$ are disjoint compact convex sets, then the sets $A^{\prime}, B^{\prime}$ indicated in Fig. 1 have the property that $\left[A^{\prime}\right] \cap\left[B^{\prime}\right]=[A] \cap[B]$.

Then (3) allows us to apply argument (2) to $A^{\prime}, B^{\prime}$. The construction in Fig. 1 has never been defined completely rigorously in the literature, to the authors' knowledge. Sylvester [25] described it as "drawing a tight string" around the sets $A, B$ with a single crossover. For further information see $[2,14,23]$.
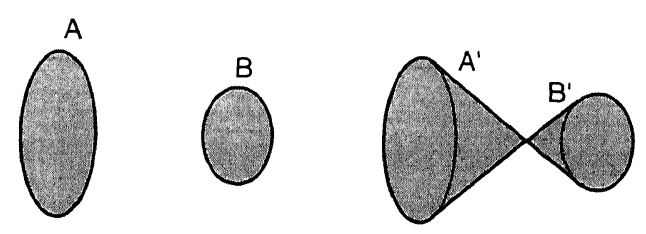

Fig. 1. Two disjoint convex sets $A, B \subset \mathbb{R}^{2}$ (left) and the Sylvester construction (right), see text 


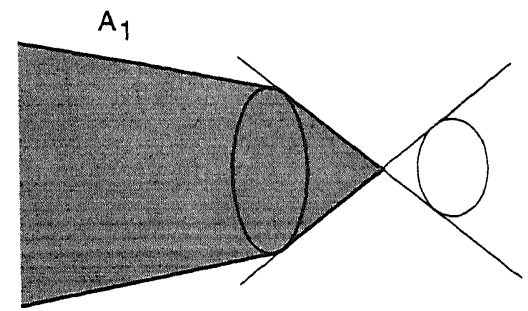

Fig. 2. The conditional closing

attice Reformulation

ıg the lattices $\mathscr{L}=\mathscr{P}\left(\mathbb{R}^{2}\right)$ and $\mathscr{M}=\mathscr{P}(\Lambda)$ define

$$
X \sim Y \Leftrightarrow \exists \ell \in Y: \ell \cap X \neq \varnothing .
$$

analogously to Sect. 5.2 we find that

$$
\begin{aligned}
\delta(X) & =[X] \\
\varepsilon(Y) & =\operatorname{spt}(Y ; \Lambda) \\
& =(\bigcup\{f \in \Lambda \mid \ell \notin Y\})^{c} \\
\varepsilon \delta(X) & =(\bigcup\{\ell \in \Lambda \mid \ell \cap X=\varnothing\})^{c}
\end{aligned}
$$

argument (1) of the previous section follows from Propositions 2-3 and the hat $\varepsilon \delta(X)=\operatorname{co} X$ for path-connected $X$. Argument (2) follows directly.

onsider argument (3). If we apply Proposition 6 with $X=A$ and $Z=B$ we find he largest set $A_{1}$ satisfying

$$
\begin{aligned}
& {\left[A_{1}\right] \cap[B]=[A] \cap[B] } \\
A_{1}= & \varepsilon\left(\delta(A) \vee \delta(B)^{*}\right) \\
= & (\bigcup\{\ell \in \Lambda \mid \ell \in[B], \quad \ell \notin[A]\})^{c}
\end{aligned}
$$

ig. 2. $A_{1}$ can also be expressed as

$$
\begin{aligned}
A_{1} & =\operatorname{spt}\left(I_{A} \vee\left(1-I_{B}\right) ; \Lambda\right) \\
& =\operatorname{spt}\left(I_{A} ;[B]\right) \\
& =\operatorname{clos}(A ;[B]),
\end{aligned}
$$

ample with the first expression indicating that $A_{1}$ is the support of the logical aation " $[B]$ implies $[A]$ ".

similar application of conditional closing to $B$ yields a set $B_{1}$ such that

$$
\left[A_{1}\right] \cap\left[B_{1}\right]=[A] \cap[B]
$$

${ }_{1}, B_{1}$ are the largest such sets.

le usual sets $A^{\prime}, B^{\prime}$ of Fig. 1 can be obtained with the same construction, by ting $\mathscr{L}$ to subsets of $\operatorname{co}(A \cup B)$; thus

$$
\begin{aligned}
& A^{\prime}=A_{1} \cap \operatorname{co}(A \cup B), \\
& B^{\prime}=B_{1} \cap \operatorname{co}(A \cup B) .
\end{aligned}
$$




\section{Practical Applications}

We now briefly indicate several other fields where the lattice formalism of Sects. 1-3 might find practical application.

\subsection{Projection Effects in Microscopy}

Let $\mathscr{L}=\mathscr{P}\left(\mathbb{R}^{3}\right), \mathscr{M}=\mathscr{P}\left(\mathbb{R}^{2}\right)$, and let $\pi: \mathbb{R}^{3} \rightarrow \mathbb{R}^{2}$ be one of the standard coordinate projections. Fix a subset $C \cong \mathbb{R}^{3}$ and define

$$
X \sim Y \Leftrightarrow \pi(X \cap C) \cap Y \neq \varnothing,
$$

equivalently

$$
X \preceq Y \Leftrightarrow \pi(X \cap C) \cong Y .
$$

This is a model for the formation of images in simple optical transmission microscopes where sets in $\mathbb{R}^{3}$ are physical objects, $C$ represents the microscope slide or physical sample of material, and $\pi$ represents the projection of light onto the image plane $\mathbb{R}^{2}$.

Then we have the mappings of Fig. 3:

$$
\delta(X)=\bigcap\{Y \mid \pi(X \cap C) \leqq Y\}=\pi(X \cap C)
$$

and

$$
\delta^{*}(X)=\bigcup\left\{Y \mid \pi\left(X^{c} \cap C\right) \leqq Y^{c}\right\}=\pi\left(X^{c} \cap C\right)^{c}
$$

which operations are known as overprojection and underprojection in microscopy $[4,5,7,6],[9$, Sect. 4, Fig. 4.1]. Overprojection occurs when the object $X$ is opaque and the surrounding medium $X^{c}$ is transparent, so that $\delta(X)$ is the dark projected image of $X$ against a light background. Underprojection occurs when $X$ is transparent and $X^{c}$ is opaque, so that $\delta^{*}(X)$ is the light image of $X$ against a dark background.

The operators $\varepsilon$ and $\varepsilon^{*}$ are two versions of the inverse projection. $\varepsilon^{*}(Y)$ is the largest subset of $C$ that will produce a given projected image $Y \leqq \mathbb{R}^{2}$. One also has
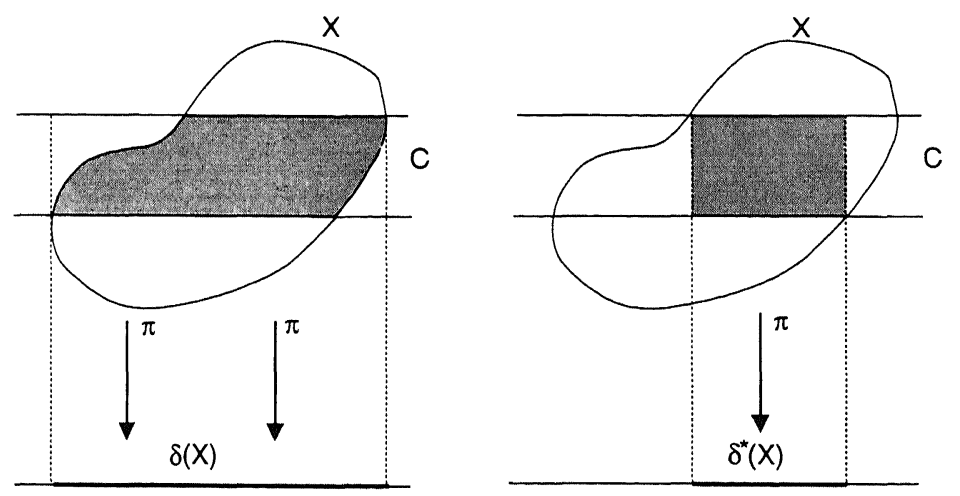

Fig. 3. Overprojection and underprojection 
the equivalence

$$
\pi(X \cap C) \cap Y \neq \varnothing \quad \text { iff } \quad X \cap \varepsilon(Y) \neq \varnothing
$$

which establishes a relationship between test sets $Y$ in the two dimensional projection and test sets $\varepsilon(Y)$ in three dimensions.

Proposition 7 states that if $X$ is known within a region $Z$ then the overprojection of $X$ is known within the underprojection of $Z$.

\subsection{Image Discretization}

A theory of image discretization must contain the following two steps. First one has to describe a sampling procedure which replaces an image in continuous space by a discrete one. Since we are restricting consideration to subsets here, this amounts to an operator mapping $\mathscr{P}\left(\mathbb{R}^{n}\right)$ into $\mathscr{P}\left(\mathbb{Z}^{n}\right)$. Secondly, in order to compare the discretised image with the original one, we must represent any set $V \cong \mathbb{Z}^{n}$ as a subset of $\mathbb{R}^{n}$.

Let $\mathscr{L}$ be the complete lattice of all closed subsets of $\mathbb{R}^{n}$ and let $\mathscr{M}$ be the complete Boolean lattice $\mathscr{P}\left(\mathbb{Z}^{n}\right)$. Let $C \subset \mathbb{R}^{n}$ be an open neighbourhood of 0 so large that copies of $C$ placed at integer positions $z \in \mathbb{Z}^{n}$ cover $\mathbb{R}^{n}$,

$$
\bigcup\left\{C_{z} \mid z \in \mathbb{Z}^{n}\right\}=\mathbb{R}^{n}
$$

Define an incidence relation $\sim$ on $\mathscr{L} \times \mathscr{M}$ by

$$
X \sim V \text { iff }(X \oplus C) \cap V \neq \varnothing .
$$

The associated adjunction $(\varepsilon, \delta)$ between $\mathscr{M}$ and $\mathscr{L}$ is given by

$$
\begin{aligned}
& \delta(X)=(X \oplus C) \cap \mathbb{Z}^{n}, \quad X \cong \mathbb{R}^{n} \text { closed }, \\
& \varepsilon(V)=\left\{x \in \mathbb{R}^{n} \mid C^{*}(x) \subseteq V\right\}, \quad V \cong \mathbb{Z}^{n} .
\end{aligned}
$$

Here $C^{*}(x)=C_{x} \cap \mathbb{Z}^{n}=\left\{z \in \mathbb{Z}^{n} \mid x \in \breve{C}_{z}\right\}$ and clearly $\varepsilon(V)$ is always closed.

In this example $\delta$ has the interpretation of a sampling operator, and $\varepsilon$ that of a representation operator. The closing $\varepsilon \delta$ can be interpreted as a reconstruction or outer approximation operator. See Fig. 4 for an illustration. The dual operators

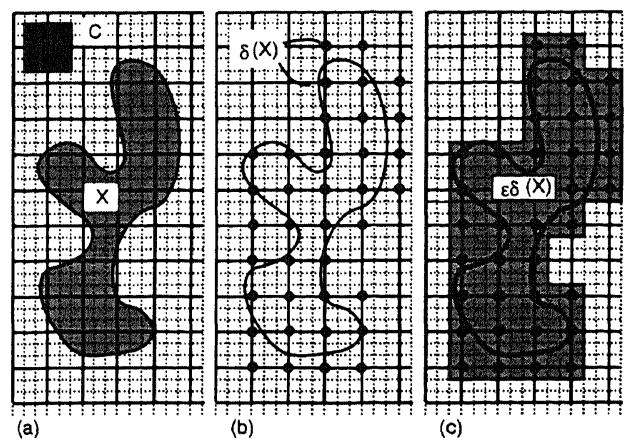

Fig. 4. (a) Original set $X$; (b) Sampled set $\delta(X)$; (c) Reconstructed set $\varepsilon \delta(X)$ 
perform similar functions on the complement of $X$, so that $\delta^{*} \varepsilon^{*}(X)$ is an inner approximation of $X$. For more details we refer to $[10,11]$.

\subsection{Robot Motion Planning}

Following [15] we describe an abstract robot as a mapping $\mathscr{A}: \mathscr{C} \rightarrow \mathscr{P}\left(\mathbb{R}^{n}\right)$ where $\mathscr{C}$ is an arbitrary space representing all possible internal states of the robot, and $\mathscr{A}(c) \subset \mathbb{R}^{n}$ is the physical position (and shape) of the robot when it is in state $c \in \mathscr{C}$.

Let $\mathscr{L}=\mathscr{P}\left(\mathbb{R}^{n}\right)$ and $\mathscr{M}=\mathscr{P}(\mathscr{C})$. Members of $\mathscr{L}$ will be called obstacles. Say that the robot in state $c$ avoids obstacle $X$ if

$$
\mathscr{A}(c) \cap X=\varnothing
$$

and otherwise $c$ hits $X$. Define an incidence relation between $\mathscr{L}$ and $\mathscr{M}$ by

$$
\begin{aligned}
& X \sim Y \Leftrightarrow \exists_{c \in Y} \mid c \text { hits } X \\
& X \preceq Y \Leftrightarrow \forall_{c \notin Y} \mid c \text { avoids } X .
\end{aligned}
$$

Then we have

$$
\begin{aligned}
\delta(X) & =\{c \in \mathscr{C} \mid c \text { hits } X\} \\
\varepsilon(Y) & =\left(\bigcup_{c \notin Y} \mathscr{A}(c)\right)^{c} \\
\delta^{*}(X) & =\{c \in \mathscr{C} \mid \mathscr{A}(c) \leqq X\} \\
\varepsilon^{*}(Y) & =\bigcup_{c \in Y} \mathscr{A}(c)
\end{aligned}
$$

The operators $\varepsilon$ and $\varepsilon^{*}$ deserve to be called support and trace respectively, since $\varepsilon(Y)$ is the region of space which must be intersected by any robot whose state belongs to $Y$, and $\varepsilon^{*}(Y)$ is the region swept out by a robot that moves through all states in $Y$.

Latombe $[15$, pp. 10,88$]$ calls $\delta(X)$ the "C-obstacle" generated by $X$ and

$$
\mathscr{C}_{\text {free }}=\bigcap_{i=1}^{m}\left(\mathscr{C} \backslash \delta\left(X_{i}\right)\right)=\mathscr{C} \backslash \delta\left(\bigcup_{i=1}^{m} X_{i}\right)
$$

the "free space" of paths avoiding obstacles $X_{1}, \ldots, X_{m}$. The robot motion planning problem can then be defined as the task of finding paths $v$ joining specified states $q_{0}, q_{1} \in C_{\text {free }}$ and satisfying $v(t) \in C_{\text {free }}$.

Latombe $[15$, p. $89 \mathrm{ff}$.] proves topological and algebraic properties of $\delta$ in the case of a 'rigid robot' where $\mathscr{C}=\mathbb{R}^{n}$ and $\mathscr{A}(c)=A_{c}$ is the translation of a fixed set $A$. Note that in this case we get $\delta(X)=X \oplus \check{A}, \varepsilon(Y)=Y \ominus \check{A}, \delta^{*}(X)=Y \ominus A$ and $\varepsilon^{*}(Y)=Y \oplus A$, so that such results can be obtained from existing results in mathematical morphology.

The local knowledge principle Proposition 7 states that (in the general case) for $X, Z \in \mathscr{L}$

$$
\delta(X) \cap \delta^{*}(Z)=\delta(X \cap Z) \cap \delta^{*}(Z)
$$

i.e. that the robot avoids an obstacle $X$ while remaining inside a space $Z$ iff it avoids $X \cap Z$ while remaining inside $Z$. The conditional closing operator can also be used to restrict attention to a subclass of permissible states of the robot. 


\section{Conclusion}

The reformulation of existing results as lattice calculations seems a trivial exercise, but has several benefits. Firstly, more-or-less-intuitive geometrical constructions are replaced by well-defined lattice operations. In the Buffon-Sylvester problem, we have obtained the first rigorous definition of Sylvester's construction (Fig. 1). Secondly, specialised 'geometrical' arguments are replaced by lattice identities, which simultaneously illuminate the structure of the problem and reduce it to trivial calculation. Thirdly, the lattice formulations of many problems are equivalent (e.g. the conditional closing operation occurs in many guises) which obviates unnecessary duplication.

Acknowledgements. We thank M. N. M. van Lieshout for thoroughly reading the manuscript. This research was supported by CWI, Amsterdam (both authors) and the University of Leiden (AJB).

\section{References}

1. Ambartzumian, R. V.: Combinatorial Integral Geometry, with applications to mathematical stereology. Chichester: John Wiley 1982

2. Baddeley, A J.: Appendix A. In: Ambartzumian R. V. Combinatorial Integral Geometry, pp. 194-214. New York Chichester: John Wiley 1982

3. Birkhoff, G.: Lattice Theory, Vol. 25 American Mathematical Society Colloquium Publications. Am. Math. Soc. Providence, RI, 3rd ed., 1984

4. Coleman, R.: The sizes of spheres from profiles in a thin slice. I. Opaque spheres. Biometrical J. 24, 273-286 (1982)

5. Coleman, R.: The sizes of spheres from profiles in a thin slice. II. Transparent spheres. Biometrical J. (1982)

6. Cruz-Orive, L. M.: On projection and truncation errors in stereology. Unpublished preprint, Department of Anatomy, University of Bern, Bern, Switzerland

7. Cruz-Orive, L. M.: Distribution-free estimation of sphere size distributions from slabs showing overprojection and truncation, with a review of previous methods. J. Microscopy 131, 265290 (1983)

8. Gierz, G., Hofmann, K. H., Keimel, K., Lawson, J. D., Mislove, M., Scott, D. S.: A compendium of continuous lattices. Berlin, Heidelberg, New York: Springer 1980

9. Gundersen, H. J. G.: Stereology of arbitrary particles. A review of unbiased number and size estimators and the presentation of some new ones, in memory of William R Thompson. $J$. Microscopy 143, 3-45 (1986)

10. Heijmans, H. J. A. M.: Discretization of morphological operators. J. Visual Commun. Image Representation 3, 182-193 (1992)

11. Heijmans, H. J. A. M.: Morphological Image Operators. Boston: Academic Press 1994

12. Heijmans H. J. A. M., Ronse, C.: The algebraic basis of mathematical morphology. Part I: dilations and erosions. Comput. Graphics Image Processing 50, 245 295 (1990)

13. Kendall, D. G.: Foundations of a theory of random sets. In: Harding, E. F., Kendall, D. G. (eds.) Stochastic geometry, Chap. 6.2, pp. 322-376. Chichester: John Wiley 1974

14. Kendall, M. G., Moran, P. A. P.: Geometrical Probability. Charles Griffin, London, 1963. Griffin's Statistical Monographs and Courses No. 10

15. Latombe, J.-C.: Robot Motion Planning. Boston, Dordrecht, London, Kluwer Academic 1991

16. Matheron, G.: Random Sets and Integral Geometry. New York: John Wiley 1975

17. Roerdink, J. B. T. M., Heijmans, H. J. A. M.: Mathematical morphology for structures without translation symmetry. Signal Processing 15, 271-277 (1988).

18. Ronse, C.: Why mathematical morphology needs complete lattices. Signal Processing, 21, $129-154(1990)$ 
19. Ronse, C., Heijmans, H. J. A. M.: The algebraic basis of mathematical morphology. Part II: openings and closings. Computer Vision, Graphics and Image Processing: Image Understanding 54, 74-97 (1991)

20. Santaló, L. A.: Integral Geometry and Geometric Probability. Encyclopedia of Mathematics and Its Applications, Vol. 1. Addison-Wesley (1976)

21. Serra, J.: Image Analysis and Mathematical Morphology. London: Academic Press 1982

22. Serra, J. (ed.) Image Analysis and Mathematical Morphology, Vol. 2: Theoretical Advances. London: Academic Press 1988

23. Solomon, H.: Geometric Probability. Number 28 in CBMS-NSF Regional Conference Series in Applied Mathematics. Society for Industrial and Applied Mathematics, Philadelphia, Pennsylvania, 1978

24. Stoyan, D., Kendall, W. S., Mecke, J.: Stochastic Geometry and its Applications. Chichester: John Wiley 1987

25. Sylvester, J. J.: On a funicular solution of Buffon's 'problem of the needle' in its most general form. Acta Mathematica 14, 185-205 (1890) 